\title{
Effects of Varying Processing Conditions on Carbon Foam Properties
}

\author{
Ping $\mathrm{ZHOU}^{1, \mathrm{a}, *}$ and Qian-Lin $\mathrm{CHEN}^{1, \mathrm{~b}}$ \\ ${ }^{1}$ College of Chemistry and Chemical Engineering, Guizhou University, Guiyang, 550025, Guizhou, \\ China \\ a 13984118220@163.com¹, b cq11018@163.com (Corresponding author email address)
}

\begin{abstract}
Purified coal-tar was chosen as the raw material for foaming precursor. Distillation methods that refining light constituent was applied as preliminary treatment of pitch. Carbon foam was prepared by the self-foaming of the resultant pitch. The effects of distilling temperature as well as time on the micro structure, bulk density, porosity, compressive strength, and thermal conductivity of carbon foam were investigated in this paper. The compressive strength of produced carbon foam can reach 6.287 $\mathrm{MPa}$ and thermal conductivity can lower than $0.048 \mathrm{~W} \cdot \mathrm{m}^{-1} \cdot \mathrm{K}^{-1}$.
\end{abstract}

\section{Introduction}

Carbon foams are regarded as a novel porous carbon material ${ }^{[1-4]}$, Such excellent physical and chemical properties such as low density, low thermal conductivity, high thermal stability, high corrosion resistance, and higher electrical conductivity are performed in these materials. The unique properties of carbon foams determine their many potential applications in numerous industries: high-temperature heat capacity materials, porous electrodes, catalyst supports, filters,structure materials, etc. ${ }^{[5-7]}$ In early 1960 s, Walter Ford acquired carbon foams from the thermal decomposition of thermoset phenolic foams. The preparation of earlier carbon foams were used organic polymer as raw material. Mesophase pitch-based carbon foams were produced by AFML(Air Force Material Laboratory), which used pitch and coal as foam precursor. At present, most of the carbon foam preparation work mainly focus on the area of use mesophase pitch as raw material. ${ }^{[8-11]}$

In the present study, almost all pitches that used as foam precursor are commercial pitches. ${ }^{[12,13]}$ Much attention has been paid to optimizing high thermal conductivity of carbon foams ${ }^{[5,14]}$, instead of its low thermal conductivity. China has large coal production, consumes large amount of coal annually. So, our country has abundant Coal-tar ${ }^{[15]}$. The content of pitch in coal tar can up to $57 \%{ }^{[16]}$. Therefore, coal tar can be used as raw material for the preparation of carbon products. Purified coal-tar was chosen as the raw material for foaming precursor. Purified coal-tar was chosen as the raw material for foaming precursor. Distillation was applied as preliminary treatment of pitch. Carbon foam was prepared by the self-foaming of the resultant pitch. The carbon foam derived from the resultant pitch possess high compressive strength and low thermal conductivity.

* Corresponding author: 13984118220@163.com 


\section{Experiment}

Coal tar used in this research was taken from a Coking Plant in Guizhou province. Impurity was conducted with simple and convenient physical and chemical experiments. The pre-treatments usually involve the polymerization/condensation of pitch by thermal treatment. The pre-treatments procedure was performed as follows. Distilling temperature was set in the range from 100 to $260^{\circ} \mathrm{C}$ and time of heat-treatment varied from $1 \mathrm{~h} \mathrm{to} 5 \mathrm{~h}$, as the methods that refining light constituent in coal tar. Then resultant samples were heated up to $350{ }^{\circ} \mathrm{C}$ at atmospheric pressure with heating rate $10{ }^{\circ} \mathrm{C} \mathrm{min}-1$ and stirred for $1 \mathrm{~h}$ in an autoclave, then heated up to the final temperature $450{ }^{\circ} \mathrm{C}$, and keeping this constant temperature for $4 \mathrm{~h}$, stirring constantly.

The particle size of foaming precursors were grounded to less than $149 \mu \mathrm{m}$. The foaming process of carbon foams was carried out in a stainless pressure vessel with the following procedures: increasing the temperature from room temperature to $480{ }^{\circ} \mathrm{C}$ at $8{ }^{\circ} \mathrm{C}$ $\min ^{-1}$, and keeping this constant temperature for $4 \mathrm{~h}$. The pressure of the autoclave was 0.8 $\mathrm{MPa}$ at room temperature.

In order to determine the porosity of the block samples both apparent and true densities were measured. The bulk density was determined by the values of the weight and the volume, the volume was calculated using dimensions of the sample. The true density was measured using standard method. The porosity was calculated using an equation:

$$
\mathrm{P}(\%)=100 \times\left(\left(\mathrm{D}_{\mathrm{t}}-\mathrm{D}_{\mathrm{b}}\right) /\left(\mathrm{D}_{\mathrm{t}}\right)\right) .
$$

where $\mathrm{P}$ - porosity; $\mathrm{D}_{\mathrm{t}}$ - true densities; $\mathrm{D}_{\mathrm{b}}$ - bulk densities.

Compressive strength was measured with cube foam samples $(2 \mathrm{~cm} \times 2 \mathrm{~cm} \times 2 \mathrm{~cm})$ by a electronic universal testing machine (WDW-10C), thermal conductivity was measured by a laser thermal conductivity testing instrument (C-Therm Tci), X-ray diffraction analyses was performed with $X^{\prime}$ Pert Powder, Micro-structure observation was measured by SEM(ZEISS).

\section{Results}

With a given distilling time of $10 \mathrm{~h}$, pitches distilled at $150{ }^{\circ} \mathrm{C}, 190^{\circ} \mathrm{C}, 230^{\circ} \mathrm{C}, 270{ }^{\circ} \mathrm{C}$, $310{ }^{\circ} \mathrm{C}$ were named as $\mathrm{P}-150, \mathrm{P}-190, \mathrm{P}-230, \mathrm{P}-270, \mathrm{P}-310$ respectively. Resultant carbon foams were named as CP-150, CP-190, CP-230, CP-270, CP-310. Similarly, distilling temperature was set as $310^{\circ} \mathrm{C}$, pitches prepared with distilling time of $2 \mathrm{~h}, 4 \mathrm{~h}, 6 \mathrm{~h}, 8 \mathrm{~h}, 10$ $\mathrm{h}$ were named as P-2, P-4, P-6, P-8, P-10. Resultant carbon foams were named as CP-2, CP-4, CP-6, CP-8, CP-10.

SEM Analysis. Fig. 1 shows the SEM images of CP-2, CP-4, CP-8. 

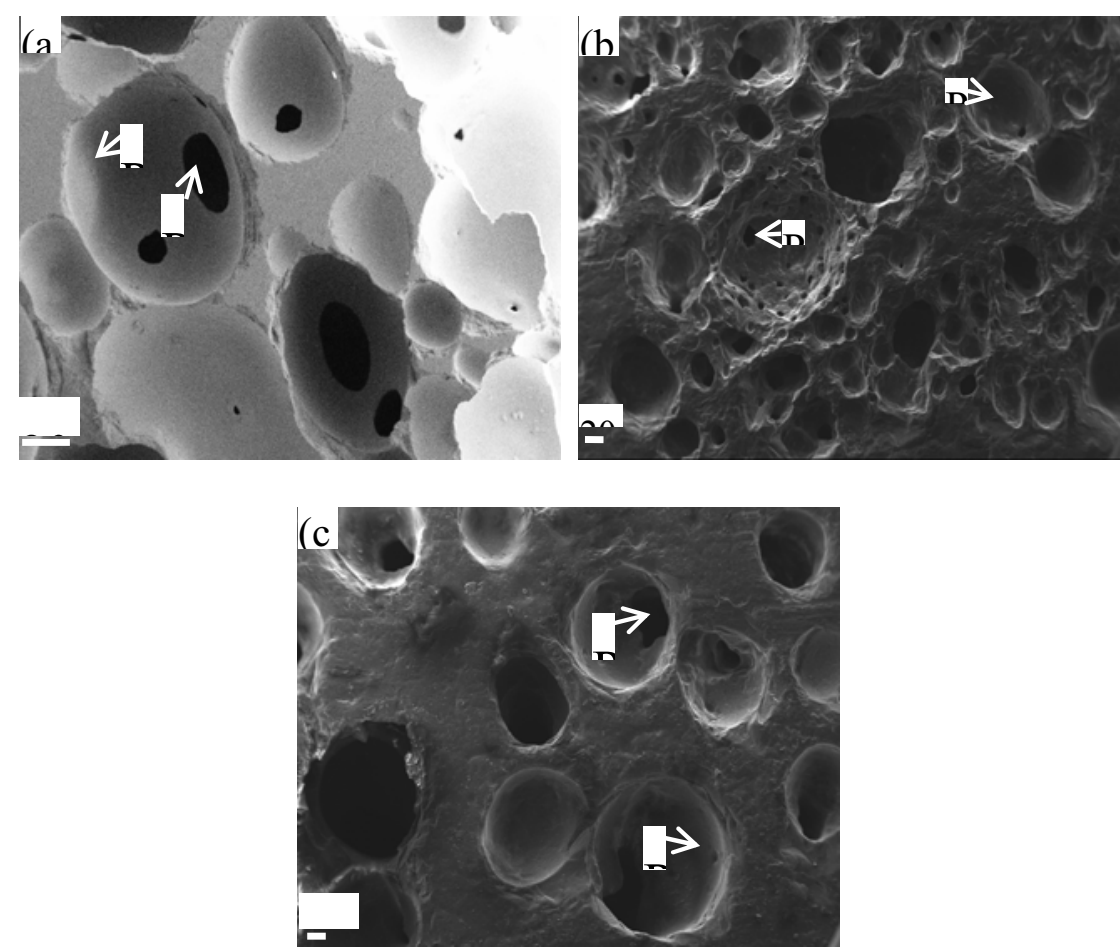

((a), (b), (c), denote CP-2, CP-4, CP-8 respectively)

Fig. 1 SEM image of carbon foam

Foam cell and ligament can be clearly seen in these foams. There are two sorts of bubbles in the samples: One of them resulted from the volatilization of the light components when heated the pitch; the other one would be the secondary bubbles, which are formed with the rupture of bubbles and further coalesce with the neighboring bubbles. The growth of bubbles generates shear force. The ligaments was produced by the stretched length of the bubble wall which caused by shear stress. The aromatic planes of the pitch arranged regularly and paralleled to the axis of ligament also caused by the shear force. Fig. 1 shows with the prolonging of distilling time, the number of bubbles and secondary bubbles in the carbon foams decreased while the width of ligaments increased obviously.

Physical Property. Fig. 2 shows the effects of distilling temperature on the physical properties of carbon foam.
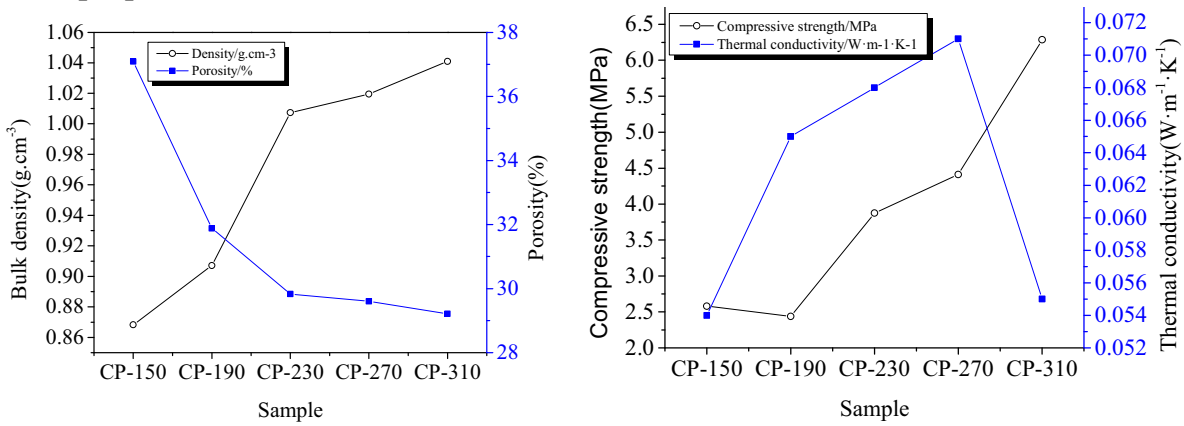

Fig. 2 the effect of distilling temperature on the physical properties of carbon foam

Fig. 2 shows that the bulk density went up with the rising distilling temperature. The 
minimum bulk density was $0.868 \mathrm{~g} \mathrm{~cm}^{-3}$ performed by CP-150. Besides, the porosity decreased with the rising distilling temperature. The compressive strength decreased first and then increased with the rising distilling temperature. The largest compressive strength was $6.287 \mathrm{MPa}$ performed by CP-310. Thermal conductivity increased first and then decreased with the rising distilling temperature. The lowest thermal conductivity was 0.054 $\mathrm{W} \cdot \mathrm{m}^{-1} \cdot \mathrm{K}^{-1}$ performed by $\mathrm{CP}-150$.

Fig. 3 shows the effects of distilling time on the physical properties of carbon foam.
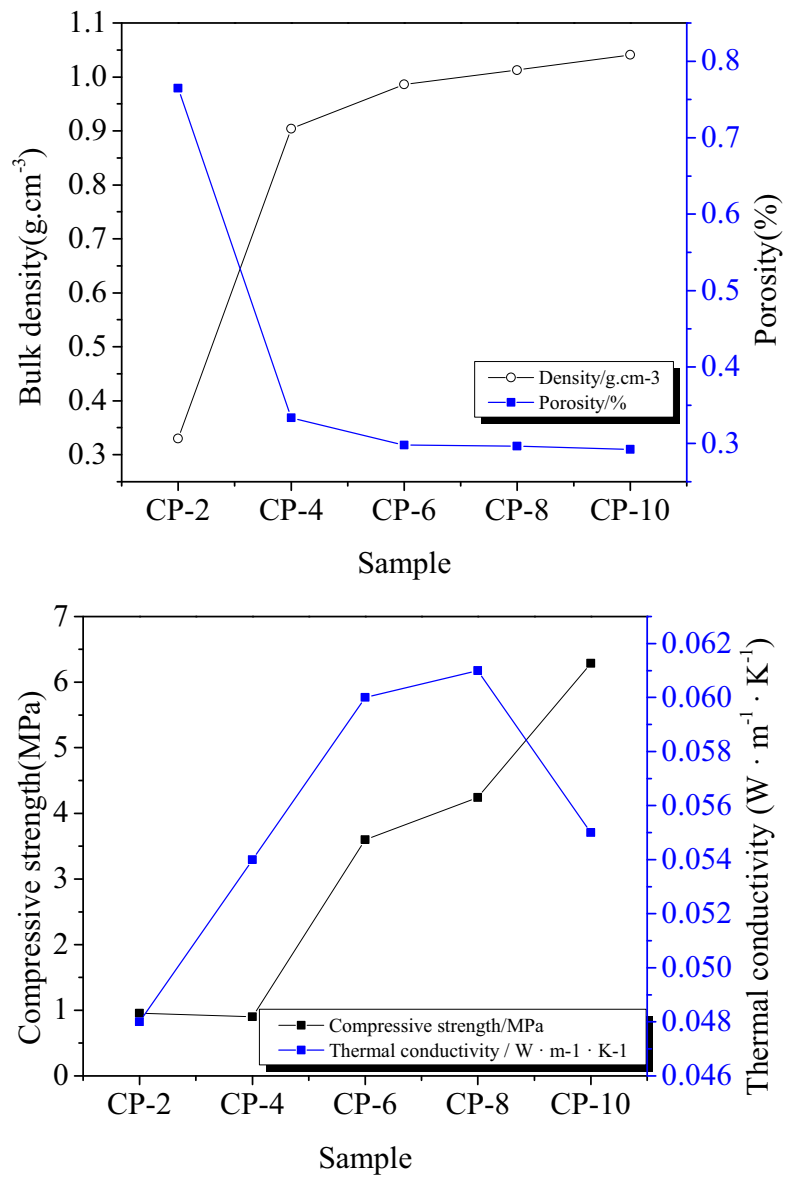

Fig. 3 The effects of distilling time on the physical properties of carbon foam

Fig. 3 shows the bulk density went up with the prolonging of distilling time. The minimum bulk density was $0.330 \mathrm{~g} \mathrm{~cm}^{-3}$ performed by $\mathrm{CP}-2$. The porosity decreased with the rising distilling time. The compressive strength decreased first and then increased with the prolonging of distilling time. The largest compressive strength was $6.287 \mathrm{MPa}$ performed by CP-10. Thermal conductivity increased first and then decreased with the prolonging of distilling time. The lowest thermal conductivity was $0.048 \mathrm{~W} \cdot \mathrm{m}^{-1} \cdot \mathrm{K}^{-}$ ${ }^{1}$ performed by CP-2.

\section{Discussion}

The content of light component in pitch decreased with the prolonging of distilling time. During foaming of the pitch, with increasing temperature, on the one hand he viscosity of 
molten pitch decreased, on the other hand the cross-linking reactions were the reason for a higher viscosity. After a long-time distilling, it was difficult for bubbles to grow up or to coalesce with each other in such an solution with large viscosity. So, bubbles and secondary bubbles in carbon foams decreased with the prolonging of distilling time. The regularly ordering of aromatic components along the the axis of ligament was promoted by surface tension with the increase of viscosity, which lead to wider ligaments.

The content of light component in pitch decreased with the rising distilling temperature. Valitilization, decomposition and condensation polymerization of light molecules in pitches were actively happening in the foaming procedure. Initially, the viscosity of pitches remained at a low level and bubbles could merge with each other easily. This is the nature of small bulk density, low strength and large porosity. Conversely, the large viscosity caused by rising distilling temperature and prolonging distilling time explained higher bulk density, higher strength and small porosity.

Bulk density of the carbon foam increased with distilling time and temperature, so as the thermal conductivity. Compared with CP-270 and CP-8, the most dense CP3-10 showed lower thermal conductivity. That is because the thermal conductivity of solid phase in CP3-10 is higher than CP-270 and CP-8. Former studies has showed that ${ }^{[17]}$, given a specific density, thermal conductivity of carbon materials is be inversely proportional to inter-planar spacing $\mathrm{d}_{002}$. Obtained foams were grounded into powder for XRD measurements, which provided data for micro structure analyzation.

Fig. 4 shows X-ray diffraction patterns of CP-310, CP-270 and CP-8. Table 1 lists the inter-plane spacing $\mathrm{d}_{002}$ of CP-310, CP-270 and CP-8. Fig. 3 and table 1 indicated that $\mathrm{d}_{002}$ in CP-310 exceeded that in CP-270 and CP-8, while CP-310 exhibited the lowest thermal conductivity among these three samples. This is in line with other researchers' findings. With a fixed bulk density, the influence of pitch processing conditions on the $\mathrm{d}_{002}$ is essential reason result in lower thermal conductivity of CP-310.

Table 1 Inter-Plane Spacing $\mathrm{D}_{002}$ In Cp-310, Cp-270 And Cp-8

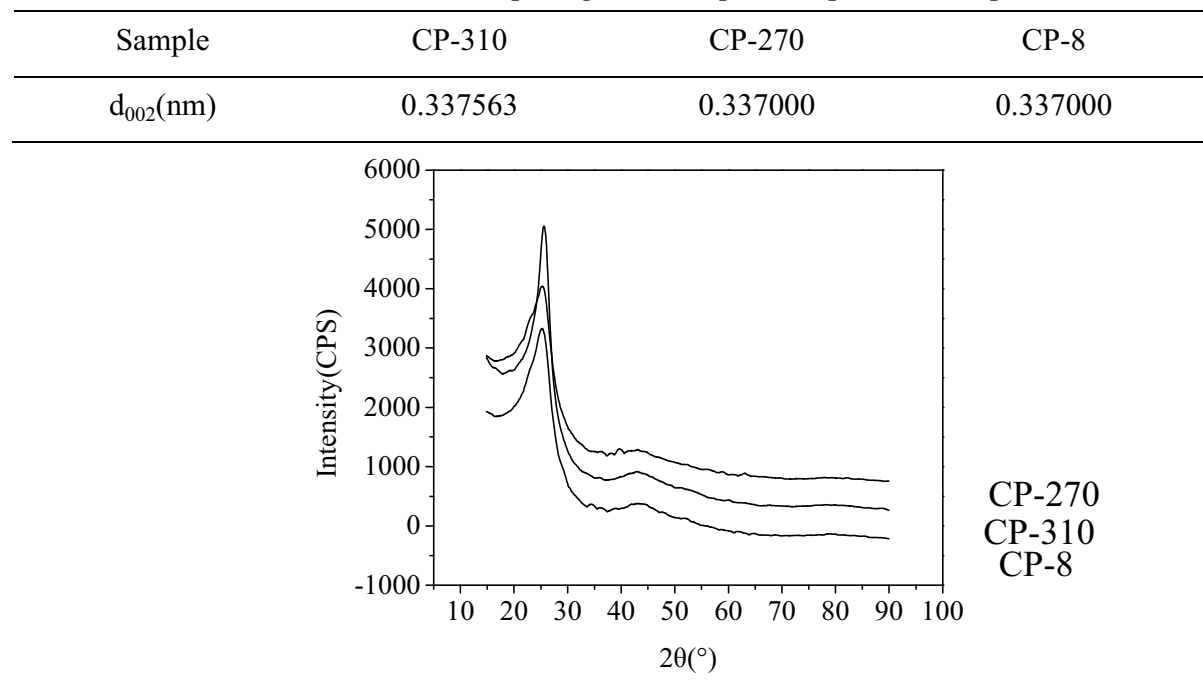

Fig. 4 X-ray diffraction patterns of CP-310, CP-270 and CP-8

\section{Summary}

In general, it was shown that such factors as temperature and time in distillation affect physical properties of foams substantially.

1. The number of pores in carbon foam decreased with distilling time while ligament 
width followed an opposite trend.

2. Bulk density, compressive strength and thermal conductivity of carbon foam increased with distilling time or temperature, with which the porosity decreased.

3. CP3-10 performed a lower thermal conductivity than CP-270 or CP-8. This could be explained as that with a fixed bulk density, $\mathrm{d}_{002}$ in carbon foams varied with pre-treatments.

\section{Acknowledgements}

The authors acknowledge the Major applied basic research project in Guizhou province (Guizhou branch in JZ word [2014]2004) and Science and technology major projects of Guizhou province (Guizhou branch [2015]6011) for their financial support.

\section{References}

1. T.Q. Li, C.Y. Wang, Preparation and Characterization of Mesophase pitch-based Carbon Foam, J. Inorg. Mater. 20(2005)1438-1444.

2. G.Z. Xu, W.W. Jin, X.Y. Zhong, S.F. Li, H.Y. Wu, Y.T. Wang, The newest development of the research for carbon foam, Mater. Rev. 28(2014)34-39.

3. C.C. Zhang, Structure Control and Application of Carbon Foams, East China Univ. (2010)1-6.

4. F. Chen, Preparation and Properties of Mesophase pitch-based Carbon Foam, Central South Univ. (2008)1-3.

5. B. Tsyntsarski, B. Petrova, T. Budinova, N. Petrov, M. Krzesinska, S. Pusz, J. Majewska, P. Tzvetkov, Carbon foam derived from pitches modified with mineral acids by a low pressure foaming process, Carbon48(2010)3523-3520.

6. N.C. Gallego, J.W. Klett, Carbon foams for thermal management, Carbon41(2003)1461-1466.

7. X.J. He, X.J. Li, X.T. Wang, N. Zhao, M.X. Yu, M.B. Wu, Efficient preparation of porous carbons from coal tar pitch for high performance supercapacitors, New Carbon Mater. 29(2014)493-502.

8. Q.X. Chen, T.H. Li, F.J. Li, Q. Zhuang, Y.L. Chen, Research Progress of Mesophase Pitch-based Carbon Foams, Carbon tech. 29(2010)28-32.

9. Z.H. Xiao, Y. Zhou, N. Xiao, J.S. Qiu, Present status and prospect of carbon foams, Chem. Ind. Eng. Prog. 27(2008)473-477.

10. X.N. Chen, The preparation and properties of carbon foam and its composite, Dong Hua Univ. (2012)1-3.

11. S.Z. Li, Y. Song, J.L. Shi, L. Liu, Q.G. Guo, Preparation and Characterization of High Strength Carbon Foam Derived From Mesophase pitch, Aerospace Mater. Tech. 6(2009)37-41.

12. S.Z. Li, Y.M. Tian, Y.J. Zhong, X. Yan, Y. Song, Formation mechanism of carbon foams derived from mesophase pitch, Carbon49(2011)618-624.

13. H.G. Liu, T.H. Li, Y.C. Shi, X.L. Wang, J. Lv, W.J. Zhang, Effect of different secondary quinoline insoluble content on the Cellular structure of carbon foam derived from coal tar pitch, J. Anal. Appl. Pyrolysis108(2014)310-315.

14. O. Mesalhy, K. Lafdi, AElgafy, Carbon foam matrices saturated with PCM for thermal protection purposes, Carbon44(2006)2080-2088.

15. J.F. Yu, X.L. Du, B.G. Wang, Purification, separation and application of coal tar, Coal Chem. Ind. (2004)29-31.

16. J.S. Gao, D.X. Zhang, J. Yu, Coal tar processing technology, Coal Chem. Ind. (2004)4-9.

17. H.P. Qiu, Q.G. Guo, Y.Z. Song, Research of the thermal conductivity and micro-crystalline of graphite material, New Carbon Mater. 17(2002)36-40. 